\title{
La tibolona disminuyó el riesgo de fractura y cáncer mamario pero aumentó el de accidente cerebrovascular
}

Tibolone reduced risk of fractures and breast cancer but increased strokes

Stevens R. y col. N Engl J Med 2008; 359:697-708.

\section{Objetivo}

Demostrar si el tratamiento con tibolona administrado en mujeres mayores con osteoporosis, reduce el riesgo de fractura vertebral y/o no vertebral, cáncer de mama y/o colorrectal, trombosis venosa profunda (TVP) o enfermedad cardiovascular.

\section{Diseño, población e intervención}

Estudio aleatorizado, doble ciego, controlado contra placebo realizado con mujeres de 60 a 85 años con una densitometría

Tabla 1: efecto de la tibolona sobre distintos eventos de interés. ósea (DMO) con un valor $\mathrm{T} \leq-2,5$ en la cadera ó columna lumbar; ó un valor $\mathrm{T} \leq-2$ y evidencia radiológica de fractura vertebral. Fueron aleatorizadas 4538 mujeres a recibir $1,25 \mathrm{mg}$ diarios de tibolona o placebo, recibiendo todas calcio y vitamina $\mathrm{D}$.

\section{Resultados principales}

Luego de una mediana de seguimiento de 34 meses, el estudio se suspendió tempranamente debido al aumento de accidentes cerebrovasculares (ACV). Los principales resultados se resumen en la tabla 1.

\begin{tabular}{|c|c|c|c|c|c|}
\hline \multicolumn{3}{|c|}{ Resultados de interés } & $\begin{array}{l}\text { Hazzard Ratio* } \\
\text { (IC95\%) }\end{array}$ & $\begin{array}{l}\text { Diferencia en el grupo } \\
\text { Tibolona (IC95\%) } \alpha\end{array}$ & NNT O NND \\
\hline \multirow[t]{4}{*}{ Fracturas } & \multirow[t]{2}{*}{ Vertebral } & Nueva & $0,55(0,41$ a 0,74$)$ & $-8,6(-12,9$ a $-4,4)$ & 116 \\
\hline & & Con fractura vertebral previa & $0,39(0,24$ a 0,63$)$ & $-20,8(-31,3$ a $-10,2)$ & 48 \\
\hline & \multirow[t]{2}{*}{ No vertebral } & Nueva & $0,74(0,58$ a 0,93$)$ & $-6,9(-12,2$ a $-1,6)$ & 144 \\
\hline & & Con fractura vertebral previa & $0,53(0,35$ a 0,81$)$ & $-17,7(-29,4$ a $-6,0)$ & 56 \\
\hline \multirow[t]{2}{*}{ Cáncer } & \multicolumn{2}{|l|}{ Mamario } & $0,32(0,13$ a 0,80$)$ & $-1,9(-3,4$ a $-0,5)$ & 526 \\
\hline & \multicolumn{2}{|l|}{ Colorrectal } & $0,31(0,10$ a 0,96$)$ & $-1,3 \quad(-2,6$ a $-0,1)$ & 769 \\
\hline \multirow[t]{3}{*}{ Enfermedad vascular } & \multicolumn{2}{|c|}{ Accidente cerebrovascular } & $2,19(1,14$ a 4,23$)$ & $2,3(0,4$ a 4,2$)$ & 434 \\
\hline & \multicolumn{2}{|c|}{ Enfermedad coronaria } & $1,37(0,77$ a 2,45$)$ & $1,1(-0,9$ a 3,2$)$ & NS \\
\hline & \multicolumn{2}{|c|}{ Tromboembolismo venoso } & $0,57(0,19$ a 1,69$)$ & $-0,6(-1,7$ a 0,5$)$ & NS \\
\hline
\end{tabular}

ACV: Accidente Cerebro Vascular. aAnualizado por cada 1000 pacientes. NNT: número necesario a tratar para evitar un evento o para producir un daño, NS: diferencia no significativa.

\section{Conclusiones}

En la mayoría de mujeres mayores con osteoporosis, y especialmente en las que ya tuvieron fracturas vertebrales, la tibolona disminuye el riesgo de nuevas fracturas; el de cáncer de mama y posiblemente el de colon. Sin embargo, su uso se asocia a un aumento de la incidencia de ACV.

Palabras clave: tibolona, terapia de reemplazo hormonal, osteoporosis.Key words: tibolone hormonal replacement therapy, osteoporosis. Fuente de financiamiento: Laboratorio Organon

\section{Comentario}

Si bien actualmente no se indica terapia de reemplazo hormonal (TRH) en forma rutinaria en la mayoría de las mujeres que consultan por síntomas o preocupaciones relacionadas al climaterio; en el caso de hacerlo, algunos especialistas prefieren inicialmente el uso de tibolona antes que estrógenos. Tienden a indicar tibolona en pacientes más jóvenes que las del presente estudio, menos preocupadas por el eventual riesgo de ACV y más atentas al riesgo eventual de cáncer de mama. Vale aclarar que en estos casos, las dosis habitualmente utilizadas suelen ser mayores (2,5mg/día) a las que se usaron en este estudio. Por el contrario, cuando las pacientes son más añosas y atendiendo a la prevención de fracturas, se tiende a utilizar tratamientos antiresortivos (bifosfonatos) en vez de TRH. Esta visión contrasta con la de los médicos de atención primaria, más conservadora con respecto a indicar TRH, especialmente luego de la difusión de los resultados de estudios como el HERS ${ }^{1}$ y el $\mathrm{WH}^{2}$. Actualmante, la indicación de TRH quedó restringida básicamente al manejo de dos situaciones: el tratamiento de los calores de la menopausia; y la prevención primaria de la osteoporosis en pacientes con menopausia precoz espontánea o quirúrgica. En ambos casos el tratamiento se ofrece por un tiempo definido -uno a tres años en el primer escenario, o

Cynthia Dreksler y Gabriel Villalón [ Servicio de Medicina Familiar y Comunitaria del Hospital Italiano de Buenos Aires.

Recibido el 07/10/2008 y aceptado el 10/11/2008

hasta llegar a los cincuenta años en el segundo ${ }^{3}$. Por el contrario, en las pacientes con osteoporosis ya establecida, la TRH ya no constituye un tratamiento de uso habitual, siendo las drogas de primera elección los bifosfonatos ${ }^{4}$. Hay que ser cautos a la hora de interpretar los resultados del presente estudio. Por un lado los autores concluyen que la tibolona reduce el riesgo de fracturas; lo que era previsible dado que es un derivado sintético hormonal. Sin embargo, el estudio fue suspendido cuando se conoció en un análisis intermedio* el aumento de riesgo de ACV, lo que coincidió con la obtención del número de pacientes y eventos necesarios para alcanzar el poder estadístico planificado que permitió demostrar diferencias estadísticamente significativas a favor de tibolona respecto del resultado primario a evaluar (la incidencia de fracturas vertebrales). Sin embargo, también es cierto que en este ensayo la tibolona mostró un efecto protector del cáncer de mama, sin aumentar el riesgo de eventos tromboembólicos.

\section{Conclusión de los comentadores}

Antes de indicar tibolona $u$ otra TRH se deberán considerar con la paciente, sus riesgos y beneficios potenciales. En lo personal, consideramos que los resultados del estudio que hemos resumido no modificarán sustancialmente el papel que cumple la TRH.

cynthia.dreksler@hospitalitaliano.org.ar ]

Ver glosario*

Dreksler C. La tibolona redujo fracturas y cáncer de mama pero aumentó los ACV. Evid. actual. práct. ambul; 11(6): 169. Nov-Dic 2008. Comentado de: Stevens R y col. The effects of Tibolone in older postmenopausal women. N Engl J Med 2008;359:697-708.PMID: 18703472. Disponible en: http://content.nejm.org/cgi/content/abstract/359/7/697 (último acceso 10/11/2008).

\section{Referencia}

1. Grady D, y col.Cardiovascular disease outcome during 6,8 years of hormone therapy. Heart and Estrogen/Progestin replacement Study (HERS II). JAMA 2002, 288:49-57. Resumido en castellano en: http://www.foroaps.org/files/TRH\%20prev\%20Primaria.pdf (último acceso 10/11/2008).

2. Rossouw J, y col. Risks and benefits of estrogen plus progestin in healthy postmenopausal women. Results of the Women's Health Initiative randomized controlled trial. JAMA 2002, 288:321-33. Resumido en castellano en: http:/www.foroaps.org/files/TRH\%20prev\%20Primaria.pdf (último acceso 10/11/2008).

3. Menopausia. En: PROFAM, Salud de la Mujer. Rubinstein E, et al Editores. 3ra edición. Buenos Aires: Fundación MF "Para el desarrollo de la Medicina Familiar y la Atención primaria de la salud", 2006. 4. Osteoporosis. En: PROFAM, Salud del Anciano. Rubinstein E, et al Editores. 3ra edición. Buenos Aires: Fundación MF "Para el desarrollo de la Medicina Familiar y la Atención primaria de la salud", 2006. 